\title{
Caveolin-1 is essential in the differentiation of human adipose-derived stem cells into hepatocyte-like cells via an MAPK pathway-dependent mechanism
}

\author{
XIN GUAN $^{1,2^{*}}$, NAN WANG ${ }^{1-3^{*}}$, FENGGONG CUI $^{2}$, YANG LIU $^{1}$, PENG LIU $^{1}$, JINGYUAN ZHAO $^{1}$, \\ CHAO HAN ${ }^{1}$, XIAOYAN LI ${ }^{1}$, ZHIQIAN LENG ${ }^{1}$, YING LI ${ }^{1}$, XIAOFEI JI ${ }^{1}$, WEI ZOU ${ }^{2}$ and JING LIU ${ }^{1}$ \\ ${ }^{1}$ Regenerative Medicine Centre, The First Affiliated Hospital of Dalian Medical University, Dalian, Liaoning 116011; \\ ${ }^{2}$ Liaoning Key Laboratories of Biotechnology and Molecular Drug Research and Development, \\ College of Life Science, Liaoning Normal University, Dalian, Liaoning 116029; \\ ${ }^{3}$ Central Laboratory, No. 6 Hospital of Dalian, Dalian, Liaoning 116031, P.R. China
}

Received December 30, 2014; Accepted September 25, 2015

DOI: $10.3892 / \mathrm{mmr} .2015 .4743$

\begin{abstract}
Human adipose-derived stem cells (hADSCs), widely present in the adult human body, are an emerging and attractive tool for the establishment of stem cell-based therapies for the treatment of liver disease. However, the mechanism underlying hADSCs hepatic differentiation remains to be elucidated. Caveolin-1 (Cav-1), a 21-24 kDa membrane structural protein, is important in liver regeneration and development. In the present study, fluorescence immunocytochemistry and western blotting were used to analyze the expression levels of Cav-1 and evaluate its effects on the hepatic differentiation of hADSCs. The results revealed that primary hADSCs preserved the ability to proliferate and differentiate into hepatocyte-like cells. As demonstrated by semiquantitative reverse transcription-polymerase chain reaction, hepatocyte-inducing factors significantly increased the expression of Cav-1 in a time-dependent manner, as indicated by increased expression levels of the albumin (ALB) and $\alpha$-fetoprotein (AFP) markers. In addition the expression levels of $A L B$ and $H N F 1 A$ significantly decreased following small interfering RNA-mediated knockdown of Cav-1. The mitogen-activated protein kinase (MAPK) signaling pathway
\end{abstract}

Correspondence to: Professor Jing Liu, Regenerative Medicine Centre, The First Affiliated Hospital of Dalian Medical University, 222 Zhongshan Road, Dalian, Liaoning 116011, P.R. China

E-mail: liujing.dlrmc@hotmail.com

Professor Wei Zou, Liaoning Key Laboratories of Biotechnology and Molecular Drug Research and Development, College of Life Science, Liaoning Normal University, 850 Huanghe Road, Dalian, Liaoning 116029, P.R. China

E-mail: weizou60@126.com

${ }^{*}$ Contributed equally

Key words: human adipose-derived stem cells, Caveolin-1, hepatic differentiation was activated during hepatic differentiation and inhibited following $\mathrm{Cav}-1$ knockdown. These results suggested that Cav-1 may regulate the hepatocyte-like differentiation of hADSCs by modulating mitogen-activated protein kinase kinase/MAPK signaling. The results of the present study will provide experimental and theoretical basis for further clinical studies on stem cell transplantation in the treatment of liver disease.

\section{Introduction}

Liver transplantation is currently the only effective therapy for end-stage liver disease and severe acute liver failure, however, its use is limited due to high costs and a shortage of allografts. Numerous studies have shown that hepatocyte transplantation may provide an alternative to liver transplantation, which is safer and more tolerable for patients suffering from end-stage liver dysfunction (1-3). However, the major limitations of using human primary hepatocytes as a cell source for transplantation are the requirement for a substantial number of cells and the low proliferative capacity of primary cells (4). Therefore, the differentiation of human stem cells into hepatocytes may provide an alternative cell source for liver-based cell therapies.

Previous in vitro studies have shown that mesenchymal stem cells are readily obtained from human bone marrow, adipose tissue and the umbilical cord, and can be differentiated into hepatocyte-like cells (5-7). Human adipose-derived stem cells (hADSCs) have been found to be a more practical candidate for autologous cell transplantation, compared with bone marrow stem cells (BMSCs) in regenerative medicine. ADSCs may be obtained repeatedly, and in large quantities through a convenient, safe and less painful procedure than that used to obtain BMSCs (8-10). Previously, ADSCs have been found to differentiate into hepatocytes in vitro and in vivo (11). Therefore, ADSCs represent a promising source of autologous adult stem cells for cell therapy in the treatment of liver disease.

The mechanism underlying the ability of ADSCs, as somatic cells, to preserve their capacity for hepatic differentiation remains to be fully elucidated. Caveolin-1 (Cav-1), a 21-24 kDa membrane structural protein, is a scaffolding protein of 
cholesterol-rich caveolae lipid rafts in the plasma membrane, which is important in cellular processes, including transport, signaling and tumor suppression $(12,13)$. It has also been implicated in the regulation of liver regeneration $(14,15)$. Cav-1 is also involved in epithelial to mesenchymal transition and in cell differentiation via the AKT, mitogen-activated protein kinase (MAPK) and transforming growth factor $\beta$-small mothers against decapentaplegic signaling pathways in pancreatic cancer cells (16). Cav-1 can also inhibit neuronal differentiation via the downregulation of the vascular endothelial growth factor, p44/42MAPK, AKT and signal transducer and activator of transcription 3 signaling pathways, thus contributing to the modulation of neuronal differentiation of neural progenitor cells or bone mesenchymal stem cells (17). Furthermore, Cav-1 is a valuable marker in the diagnosis of rhabdomyosarcoma, as these cells exhibit a low degree of differentiation and a mechanical association to the extracellular signal-regulated kinase/MAPK signaling pathways (18). Whether Cav-1 is involved in the differentiation of hADSCs into hepatic cells remains to be elucidated, as do the signal transduction pathways underlying these effects.

The present study aimed to determine whether Cav-1 is involved in the differentiation of hADSCs into hepatic cells and to dissect the signal transduction pathways underlying these effects. This was investigated by initially separating and identifying hADSCs from human adipose tissue, followed by assessing the effects of Cav-1 through analysis of the signal transduction pathways involved in the process of hADSC differentiation into hepatocytes. Cav-1 and its associated signaling pathway appear promising for use in stem cell-based clinical applications of the treatment of liver cancer.

\section{Materials and methods}

Isolation and culture of hADSCs. Human adipose tissue was obtained from five healthy donors (two male and three female; age, 20-45 years; $10 \mathrm{ml}$ tissue samples/individual) at the First Affiliated Hospital of Dalian Medical University (Dalian, China), and the study was approved by the ethics committee of the First Affiliated Hospital of Dalian Medical University. Written informed consent was obtained from the donors. The hADSCs were isolated from raw liposuction aspirate, as described previously (19). Briefly, the raw liposuction aspirate was washed extensively three times with equal volumes $(10 \mathrm{ml})$ of sterile phosphate-buffered saline (PBS) containing $1 \%$ penicillin and streptomycin (Gibco; Thermo Fisher Scientific, Inc., Waltham, MA, USA). The extracellular matrix was digested with $1 \%$ type I collagenase (Worthington Biochemical Corporation, Lakewood, NJ, USA) at $37^{\circ} \mathrm{C}$ for $60 \mathrm{~min}$. The cells were separated through a $70 \mu \mathrm{m}$ mesh filter (BD Biosciences, San Jose, CA, USA) and the cell sap was centrifuged at $180 \mathrm{x}$ g for $5 \mathrm{~min}$. The hADSC pellet was suspended in Dulbecco's modified Eagle's medium (Gibco; Thermo Fisher Scientific, Inc.) containing 10\% fetal bovine serum (Gibco; Thermo Fisher Scientific, Inc.) and maintained at $37^{\circ} \mathrm{C}$ in $5 \% \mathrm{CO}_{2}$ (all incubation conditions for regular cell culture in this study were $37^{\circ} \mathrm{C}$ in $5 \% \mathrm{CO}_{2}$ ). Cell cultures at a confluence of $80-90 \%$ were used for subsequent experiments. To quantify cell growth, the hADSCs were seeded in triplicate at a density of 4,000 cells per well in 24-well plates.
The cells were digested and counted using a hemocytometer (XB-K-25; Anxin Co., Shanghai, China) every day for 7 days. Three wells were used for each cell count and the analysis was repeated in triplicate.

Fluorescence immunocytochemistry. The hADSCs were seeded at a density of 4,000 cells/well in 24-well plates. The cells were then rinsed with PBS three times and fixed in $4 \%$ paraformaldehyde (Tianjin Bodi Chemical Co., Ltd., Tianjin, China) at room temperature for $1 \mathrm{~h}$. Following washing, $2 \mathrm{ml}$ PBS mixed with 1\% Triton X-100 (Beijing Solarbio Science \& Technology Co., Ltd., Beijing, China) was added to each well for $10 \mathrm{~min}$ to increase the permeability of the cell membranes. The samples were incubated with mouse monoclonal anti-human Cav-1 antibody (1:100; cat. no. sc-53564; Santa Cruz Biotechnology, Inc., Dallas, TX, USA), mouse monoclonal anti-human albumin (ALB; 1:70; cat. no. sc-271604; Santa Cruz Biotechnology, Inc.), mouse monoclonal anti-human $\alpha$-fetoprotein (AFP; 1:70; cat. no. sc-166335; Santa Cruz Biotechnology, Inc.) at $4^{\circ} \mathrm{C}$ overnight. Following washing three times with PBS, FITC-labeled goat anti-mouse immunoglobulin (Ig)G (1:70; cat. no. F0382; Sigma-Aldrich, St. Louis, MO, USA) was added for $1 \mathrm{~h}$ at room temperature, followed by washing with PBS. Hoechst 33258 (1:500; Sigma-Aldrich) was then added for nuclear staining.

Flow cytometric analysis. Flow cytometry (BD FACSAria II; BD Pharmingen, San Diego, CA, USA) was performed on the hADSCs. The cells were washed twice with PBS and counted under a microscope (Leica Microsystems GmbH, Wetzlar, Germany). Cells were analyzed using conjugated mouse monoclonal antibodies. The cells were washed once in flow wash buffer ( $1 \mathrm{X}$ Dulbecco's BPS, $0.5 \%$ bovine serum albumin and $0.1 \%$ sodium azide), resuspended in blocking buffer (wash buffer with $25 \mu \mathrm{g} / \mathrm{ml}$ mouse IgG), and incubated for $10 \mathrm{~min}$ at room temperature. A total of $100 \mu \mathrm{l}$ cell suspension $\left(\sim 1 \times 10^{5}\right.$ cells) was added to each tube, and labeled monoclonal antibodies were added for tricolor analysis (FITC and PE). Isotype control combinations were performed to reflect monoclonal isotype combinations. The following antibodies: Monoclonal mouse anti-human CD13 PE (cat. no. 347837), CD34 PE (cat. no. 348057), CD45 FITC (cat. no. 347463), CD71 FITC (cat. no. 347513), CD90 FITC (cat. no. 555595) and CD106 PE (cat. no. 555647) were purchased from BD Pharmingen. CD90 was diluted at 1:100 and the remaining antibodies were diluted at 1:10. All tubes were incubated and protected from the light for $30 \mathrm{~min}$. Following incubation, the cells were washed once with wash buffer and fixed in $200 \mu \mathrm{l}$ $4 \%$ paraformaldehyde. Data analysis was performed using BD FACSDiva Software v 6.1.3 (BD Pharmingen).

Adipogenic differentiation of $h A D S C s$. For adipogenic differentiation, the hADSCs were seeded into a 6-well plate at $1 \times 10^{4}$ cells per well in complete medium. Following $24 \mathrm{~h}$ incubation, the cells were cultured in AdipoDiff medium, comprising H-DMEM with 10\% FBS, 0.5 mmol/1 1,3-isobutyl methylxanthine (IBMX; Sigma-Aldrich), $1 \times 10^{-6} \mathrm{M}$ dexamethasone (Dex; Sigma-Aldrich), $10 \mu \mathrm{g} / \mathrm{ml}$ insulin (Sigma-Aldrich) and $0.2 \mathrm{mmol} / 1$ indometacin (Sigma-Aldrich), for 2 weeks, with medium replaced with fresh medium every 3 days. The 
Table I. List of primer sequences used for semiquantitative reverse transcription-polymerase chain reaction analysis.

\begin{tabular}{|c|c|}
\hline Gene (Accession; length) & Primer sequence \\
\hline Oct (NM_001173531.2; 433 bp) & $\begin{array}{l}\text { Forward 5'-GGGGTTCTATTTGGGAAGGTATT-3' } \\
\text { Reverse 5'-CAGAGTGGTGACGGAGACAGG-3' }\end{array}$ \\
\hline Nanog (NM_024865.2; 367 bp) & $\begin{array}{l}\text { Forward 5'-ACCTATGCCTGTGATTTGTGGG-3' } \\
\text { Reverse 5'-GGGTTGTTTGCCTTTGGGAC-3' }\end{array}$ \\
\hline$A L B$ (NM_000477.5; 598 bp) & $\begin{array}{l}\text { Forward 5'-GTGTTGATTGCCTTTGCTCAGTAT-3' } \\
\text { Reverse 5'-GGAGGTTTGGGTTGTCATCTTTGT-3 }\end{array}$ \\
\hline AFP (NM_001134.2; 174 bp) & $\begin{array}{l}\text { Forward 5'-ATTGAGAAACCCACTGGAGATGA-3 } \\
\text { Reverse 5'-TAGCGAGCAGCCCAAAGAAG-3' }\end{array}$ \\
\hline CYP1A1 (NM_000499.3; 147 bp) & $\begin{array}{l}\text { Forward 5'-TCCCTATTCTTCGCTACCTACCC-3' } \\
\text { Reverse 5'-TCAGGCTGTCTGTGATGTCCC-3' }\end{array}$ \\
\hline HNF1A (NM_000545.5; 208 bp) & $\begin{array}{l}\text { Forward 5'-GAGGACGAGACGGACGACGAT-3' } \\
\text { Reverse 5'-GGAGTGCCCTTGTTGAGGTGTT-3' }\end{array}$ \\
\hline HNF1B (NM_000458.2; 152 bp) & $\begin{array}{l}\text { Forward 5'-CCCCTATGAAGACCCAGAAGC-3' } \\
\text { Reverse 5'-GACACGGACCTCAGTGACCAA-3' }\end{array}$ \\
\hline GAPDH (NM_001256799.2; 700 bp) & $\begin{array}{l}\text { Forward 5'-TGACCTGCCGTCTAGAAAACC-3' } \\
\text { Reverse 5'-GTGGGTGTCGCTGTTGAAGTC-3' }\end{array}$ \\
\hline
\end{tabular}

ALB, albumin; AFP, $\alpha$-fetoprotein; Oct4, octamer-binding transcription factor 4; CYP1A1, cytochrome P450, family 1, member A1; HNF1A hepatocyte nuclear factor-1- $\alpha$; HN1B, hepatocyte nuclear factor-1- $\beta$; GAPDH, glyceraldehyde 3-phosphate dehydrogenase.

induction of adipogenic differentiation was detected using Oil Red O staining (Sigma-Aldrich). The formalin fixed cells were washed three times with PBS and stained with $0.5 \%$ Oil Red $\mathrm{O}$ for $5 \mathrm{~min}$. Following counterstaining with hematoxylin (Beyotime Institute of Biotechnology, Shanghai, China), the red colored lipids were detected under a light microscope (DMI4000B; Leica Microsystems GmbH).

Osteogenic differentiation of hADSCs. For osteogenic differentiation, the hADSCs were seeded into a 6-well plate at $1 \times 10^{4}$ cells per well in complete medium for $24 \mathrm{~h}$. Subsequently, OsteoDiff medium was added (H-DMEM with $10 \% \mathrm{FBS}, 1 \times 10^{-7}$ M Dex, $10 \mathrm{mmol} / \mathrm{l} \beta$-sodium glycerophosphate and $50 \mu \mathrm{mol} / \mathrm{l}$ vitamin C; both from Sigma-Aldrich) to induce osteogenesis for 3 weeks. The osteogenic differentiation was evaluated by the calcium accumulation using Von Kossa staining. Briefly, the cells were rinsed with PBS and fixed with $95 \%$ ethanol at room temperature for $10 \mathrm{~min}$. Following treatment with $2 \%$ silver nitrate (Tianjin Bodi Chemical Co., Ltd.) and exposure to sunlight for 15-60 min, the cells were washed and then reduced by $2 \%$ sodium thiosulfate (Tianjin Bodi Chemical Co., Ltd. ) for $1 \mathrm{~h}$. Eosin (Beyotime Institute of Biotechnology) was used for counterstaining, and the cells were observed using a microscope (Leica DMI4000B).

Hepatic induction. Passage four cells were seeded into 6-well plates at a density of 8,000 cells per well in complete medium for $24 \mathrm{~h}$. The complete medium was replaced with serum-free H-DMEM with $10 \mathrm{ng} / \mathrm{ml}$ basic fibroblast growth factor (bFGF; PeproTech, Inc., Rocky Hill, NJ) and $20 \mathrm{ng} / \mathrm{ml}$ human epidermal growth factor (PeproTech, Inc.) for $48 \mathrm{~h}$. The cells were then cultured in H-DMEM with $10 \mathrm{ng} / \mathrm{ml} \mathrm{bFGF}, 20 \mathrm{ng} / \mathrm{ml}$ hepatocye growth factor (Sigma-Aldrich), $15 \% \mathrm{FBS}$ and $1 \times 10^{-7} \mathrm{M}$ Dex for 7,14 and 21 days.

ELISA analysis. Culture supernatants were collected for the measurement of urea content using an ELISA kit (cat. no. MAK077; Sigma-Aldrich) according to the manufacturer's instructions. Absorbance was read at $450 \mathrm{~nm}$ using an ELx808 Absorbance Reader (Bio-Tek Instruments, Inc., Winooski, VT, USA) and analyzed using Gen5 software (Bio-Tek Instruments, Inc.).

Transient transfection of Cav-1 siRNA into hADSCs. For transfection, $8 \times 10^{3}$ cells per well were seeded into 6 -well plates for $24 \mathrm{~h}$ and induced towards hepatic differentiation. $2 \mu \mathrm{g} \mathrm{Cav}-1$ siRNA was transiently transfected into the cells on days 5 and 12 using Lipofectmine 2000 (Invitrogen; Thermo Fisher Scientific, Inc.), according to the manufacturer's protocol. The cells were incubated in compete medium $4 \mathrm{~h}$ following transfection and were collected for examination after $48 \mathrm{~h}$.

Semiquantitative reverse transcription-polymerase chain reaction $(R T-P C R)$. Total RNA was extracted from the cells using RNAiso Plus (Takara Bio, Inc., Otsu, Japan), according to the manufacturer's protocol. RNA (5 $\mu \mathrm{g})$ was reverse transcribed using MMLV Reverse Transcriptase (Takara Bio, Inc.) and oligo dT. The primers (Takara Bio, Inc.) used for RT-PCR are listed in Table I. A PCR kit (Premix Taq ${ }^{\mathrm{TM}}$; cat. no. RR901A; Takara Bio, Inc.) was used according to the manufacturer's instructions. The reaction system contained cDNA $(2.5 \mu \mathrm{l})$, Premix Taq $(25 \mu \mathrm{l})$, primers $(2 \mu \mathrm{l})$ and sterile purified water $(\leq 50 \mu \mathrm{l})$. The samples were amplified with an initial denaturation step of $94^{\circ} \mathrm{C}$ for $5 \mathrm{~min}$, followed by 30 cycles 
A
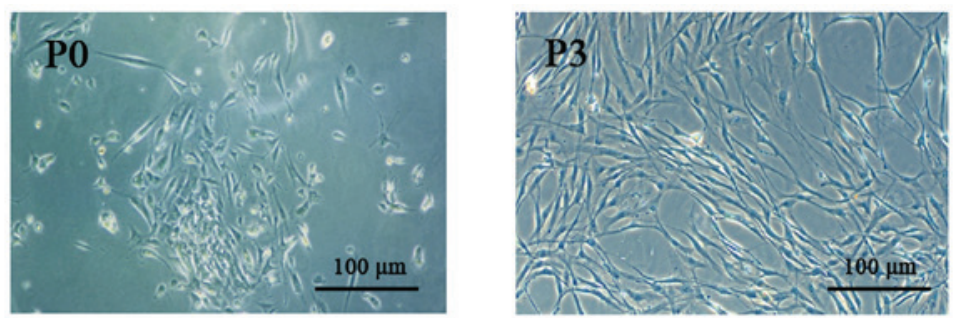

B
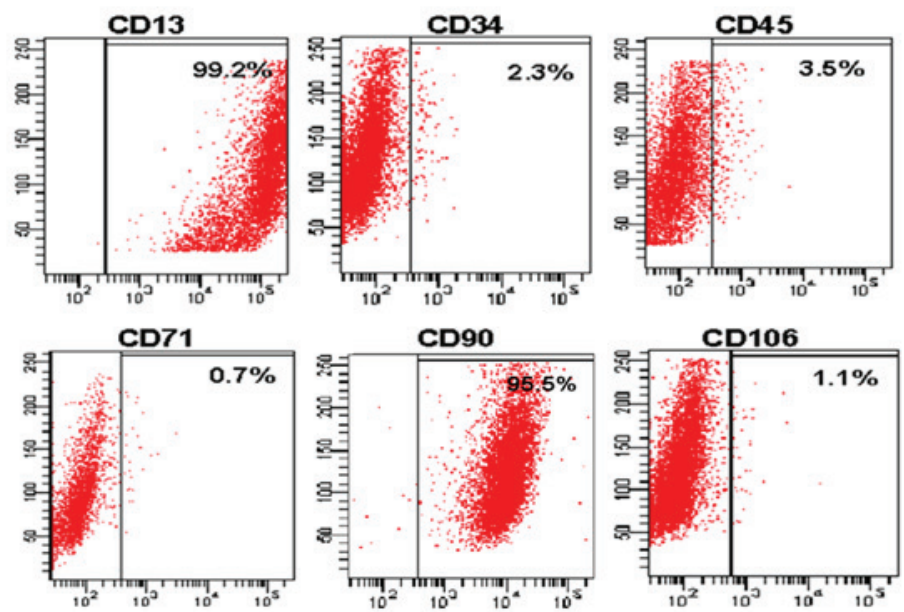

C

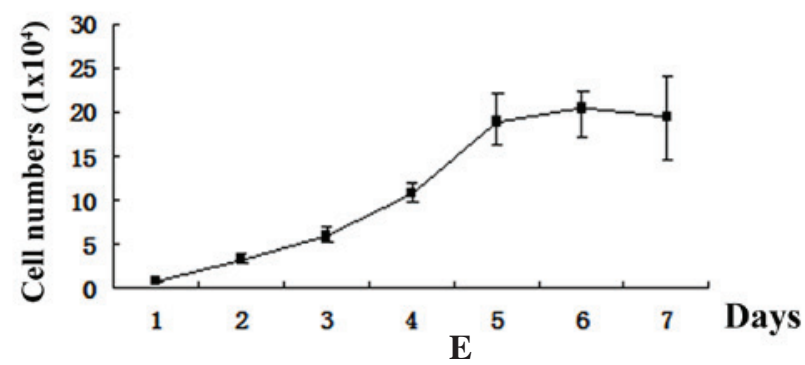

D
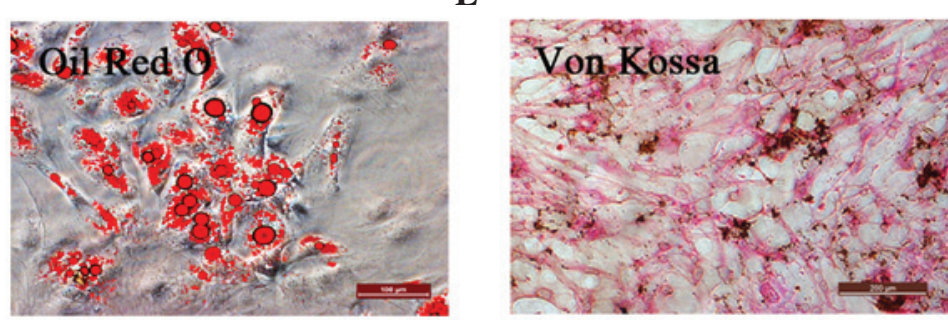

Figure 1. Isolation and characteristic confirmation of hADSCs. (A) Morphology of hADSCs. P3 hADSCs were used for subsequent experiments (scale $\mathrm{bar}=100 \mu \mathrm{m}$ ). (B) Flow cytometry detection of ADSC surface markers. Fluorescence intensity was associated with the degree of expression. The percentages indicated the positive rates of ADSC surface markers detected by flow cytometry analysis. (C) hADSC proliferation was evaluated by counting the numbers of cells each day for 1 week. Data are expressed as the mean \pm standard deviation. (D) Oil Red O staining of the adipogenic-differentiated hADSCs, with the red circles representing lipid droplets within the differentiation (scale bar $=200 \mu \mathrm{m}$ ). (E) Von Kossa staining of the osteogenic-differentiated hADSCs (scale bar $=200 \mu \mathrm{m})(\mathrm{n} \geq 3)$. hADSCs, human adipose-derived stem cells; P0, primary hADSC cultures; P3, third generation hADSC cultures.

of $94^{\circ} \mathrm{C}$ for $30 \mathrm{sec}, 55^{\circ} \mathrm{C}$ for $30 \mathrm{sec}, 72^{\circ} \mathrm{C}$ for $1 \mathrm{~min}$ and a final extension step of $72^{\circ} \mathrm{C}$ for $10 \mathrm{~min}$, using a PCR thermocycler (Techne TC-512; Bibby Scientific Ltd., Staffordshire, UK). The PCR products $(15 \mu \mathrm{l})$ were separated by electrophoresis on $1 \%$ agarose gels (Invitrogen; Thermo Fisher Scientific, Inc.) and visualized with ethidium bromide (Sigma-Aldrich; final concentration $0.5 \mu \mathrm{g} / \mathrm{ml}$ ) staining under ultraviolet illumination (24-25-PR; DNR Bio-Imaging Systems Ltd., Jerusalem, Israel).

Western blot analysis. The cells were disrupted with radioimmunoprecipitation assay buffer (Beyotime Institute of Biotechnology), containing $50 \mathrm{mM}$ Tris- $\mathrm{HCl}$ ( $\mathrm{pH} 8.0$ ),
$150 \mathrm{mM} \mathrm{NaCl}, 0.25 \mathrm{mM}$ EDTA (pH 8.0), 0.1\% SDS, $1 \%$ Triton X-100, $50 \mathrm{mM} \mathrm{NaF}$ and a protease inhibitor cocktail. The lysates were boiled in gel-loading buffer (Takara Bio, Inc.) and separated on 10\% SDS-PAGE gels (Beijing Solarbio Science \& Technology Co., Ltd.). Following PAGE, the proteins were transferred onto a polyvinylidene fluoride membrane (EMD Millipore, Billerica, MA, USA). The membrane was blocked with 5\% non-fat dry milk (dissolved by PBS with Tween 20; Gibco; Thermo Fisher Scientific, Inc.) at room temperature for $1 \mathrm{~h}$, prior to being probed with the indicated antibodies and visualized with horseradish peroxidase-conjugated polyclonal goat antimouse (1:5,000; cat. no. 31430; Thermo Fisher Scientific, 
A

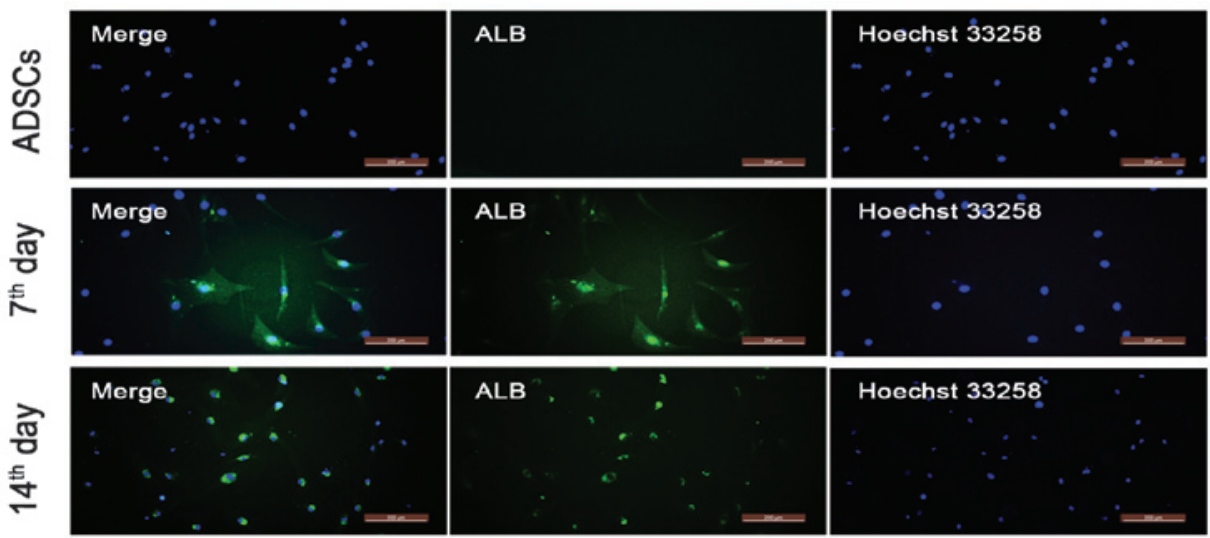

B
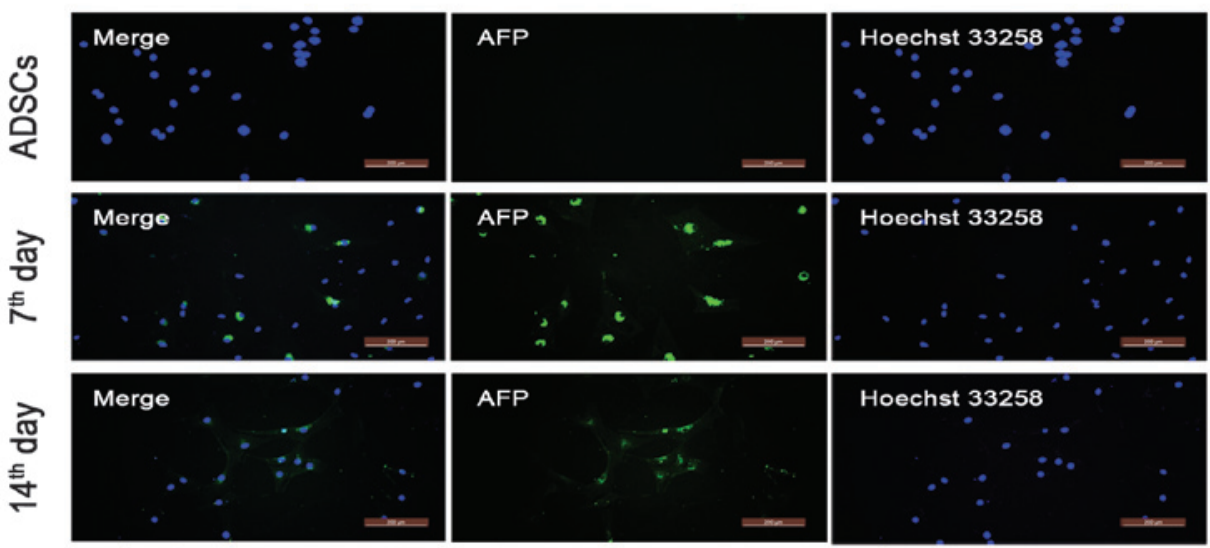

C

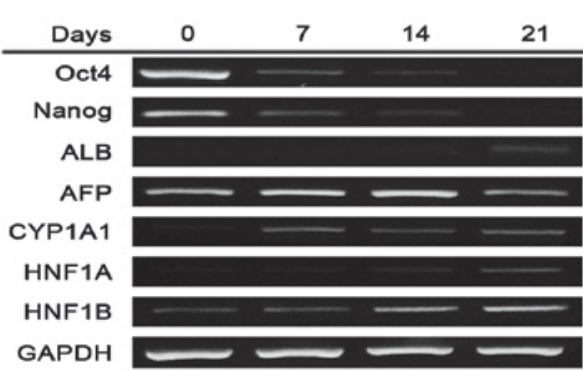

D

Figure 2. Isolated hADSCs retain hepatic differentiation capacity. Expression levels of (A) ALB and (B) AFP were detected using immunofluorescence following 7 and 14 days of hADSC differentiation. The nuclei were stained with Hoechst (right panel) and the target proteins (ALB and AFP) were stained with relative antibodies (middle panel). The merged images are shown in the left panel (scale bar=200 $\mu \mathrm{m}$ ). (C) Stem cell- and hepatocyte-specific gene transcription was detected using semiquantitative reverse transcription-polymerase chain reaction analysis following 7, 14 and 21 days of hADSC differentiation. (D) Urea content in the culture medium of the induction group. Supernatants were collected and stored at $-20^{\circ} \mathrm{C}$. For detection, samples were processed according to the manufacturer's protocol, and the optical density was detected using a spectrometer at $450 \mathrm{~nm}$ within $15 \mathrm{~min}$ following the addition of stop buffer. " $\mathrm{P}<0.05 \mathrm{vs}$. untreated cells; ${ }^{* *} \mathrm{P}<0.01$ vs. untreated cells $(\mathrm{n} \geq 3$ ). hADSCs, human adipose-derived stem cells; ALB, albumin; AFP, $\alpha$-fetoprotein; Oct4, octamer-binding transcription factor 4; CYP1A1, cytochrome P450, family 1, member A1; HNF1A hepatocyte nuclear factor-1- $\alpha$; HNF1B, hepatocyte nuclear factor-1- $\beta$; GAPDH, glyceraldehyde 3-phosphate dehydrogenase.

Inc.) and polyclonal goat anti-rabbit (1:5,000; cat. no. 31460; Thermo Fisher Scientific, Inc.) secondary antibodies and enhanced chemiluminescence reagents (GE Healthcare Life Sciences, Piscataway, NJ, USA). The phosphorylated monoclonal mouse anti-human (p)-MAPK (1:2,000; cat. no. 9106) and polyclonal rabbit anti-human total MAPK $(1: 1,000$; cat. no. 9102) antibodies were purchased from Cell Signaling Technology, Inc.

Statistical analysis. All values are expressed as the mean \pm standard deviation. Student's t-test was used to identify significant differences using SPSS 14.0 software (SPSS,
Inc., Chicago, IL, USA). $\mathrm{P}<0.05$ was considered to indicate a statistically significant difference.

\section{Results}

hADSCs isolation and characteristic identification. The present study isolated hADSCs from adipose tissue (Fig. 1A). The cells began to attach to flasks and proliferate to passage three. To determine whether the isolated cells preserved ADSC characteristics, the expression levels of the CD13 and CD90 stem cell surface markers were quantified and were found to be 99.2 and 95.5\%, respectively. CD34, CD45, CD71 and CD106 were not 
A

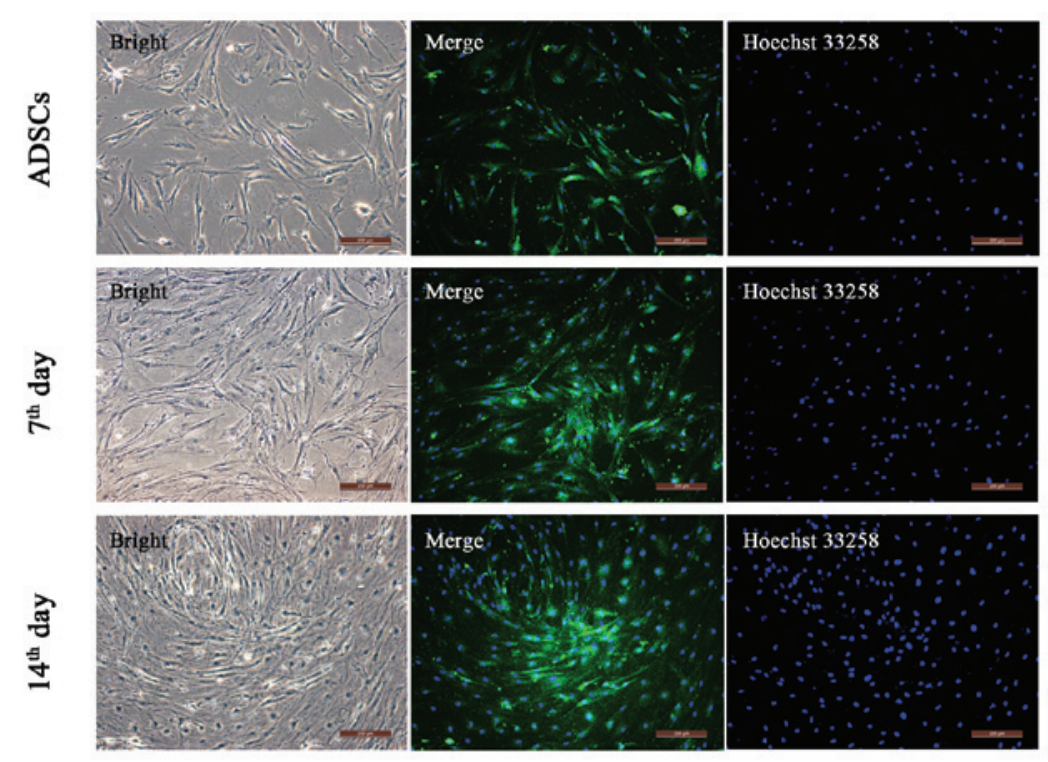

B
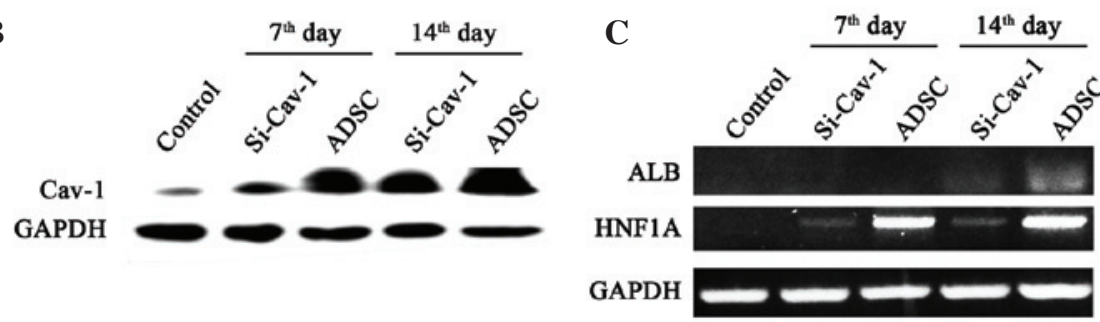

D

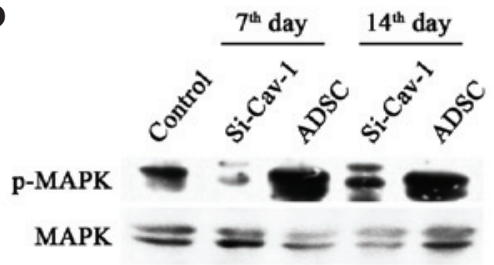

Figure 3. Effects of Cav-1 on ADSC developmental stages. (A) Immunofluorescence staining of undifferentiated hADSCs (upper), differentiated for 7 days (middle) and 14 days (lower). All samples were detected under fluorescence microscopy, and the merged images of Cav-1 (stained green) and nuclei (stained blue) are shown in the middle (Scale $\mathrm{ba}=200 \mu \mathrm{m}$ ). (B) Western blot analysis of Cav-1 in undifferentiated (control) and differentiated hADSCs for 7 and 14 days. (C) Semiquantitative reverse transcription-polymerase chain reaction analyses of $A L B$ and $H N F 1 A$ in differentiated and normal hADSCs. (D) Western blot analysis of the expression levels of p-MAPK and MAPK in differentiated and normal hADSCs. hADSCs, human adipose-derived stem cells; Cav-1, Caveolin-1; si, small interfering RNA; MAPK, mitogen-activated protein kinase; p-phosphorylated; GAPDH, glyceraldehyde 3-phosphate dehydrogenase.

expressed. This indicated that isolated hADSCs remained in an undifferentiated state (Fig. 1B). The results of the proliferation assay showed that the isolated hADSCs were capable of proliferation, an essential property of ADSCs (20) (Fig. 1C).

Two weeks following exposure to AdipoDiff medium, intracellular lipid droplets were observed within the adipogenic-differentiated hADSCs using Oil Red O staining (Fig. 1D). Von Kossa staining of the hADSCs was maintained in the OsteoDiff medium during differentiation (Fig. 1E).

Hepatic differentiation capacity of hADSCs. To evaluate whether isolated and primary cultured hADSCs retained hepatic differentiation ability, hepatocyte inducing factors were added, and hepatocyte marker proteins and genes were detected. $\alpha$-fetoprotein (AFP) and albumin (ALB) were expressed at high levels on days 7 and 14, but were undetectable in the untreated hADSCs, indicating that the hADSCs underwent successful hepatic differentiation (Fig. 2A and B). To confirm this, semiquantitative RT-PCR was performed to detect markers of gene transcription. The ADSC marker genes, Oct4 and Nanog, were activated in the absence of induction, and were gradually inactivated as the hADSCs differentiated into hepatocytes. Oct4 and Nanog were almost undetectable on day 21, indicating the complete differentiation of hADSCs into hepatocytes (Fig. 2C). The present study also assessed the hepatic marker genes, $A L B$, CYPIA1, HNF1A and HNF1B, which gradually increased and were most prevalent on day 21. AFP, a gene associated with the major plasma protein produced by the yolk sac and liver during fetal development, decreased as hADSCs underwent differentiation, which was in accordance with liver development (Fig. 2C). Additionally, urea synthesis, known as an important function of hepatocytes, significantly increased with the duration of hepatic differentiation (Fig. 2D). These results suggested that isolated hADSCs differentiated into hepatocyte-like cells. 
Downregulation of Cav-1 inhibits hepatic marker proteins by attenuating the MEK/MAPK pathway during the induction of hepatocyte-like cells from hADSCs. On days 7 and 14 of differentiation, the results of the immunostaining and western blot analyses showed that the expression of Cav-1 was significantly increased, compared with that in the control, indicating a positive role in hepatocyte differentiation (Fig. 3A and B). To assess whether Cav-1 has a central role in hepatic differentiation, the present study transiently knocked down Cav-1 on days 5 and 12 (Fig. 3B). With the suppression of Cav-1, the gene expression levels of $A L B$ and $H N F 1 A$ were significantly reduced (Fig. 3C), indicating attenuation of the hepatic differentiation process following $C a v-1$ knockdown. Furthermore, to identify the signaling pathway involved in differentiation, the present study examined the MAPK pathway and found that it was significantly activated during early differentiation. In addition, the MAPK pathway was largely attenuated following Cav-1 knockdown (Fig. 3D).

\section{Discussion}

hADSCs are widely present in the adult human body and have been suggested as an emerging and attractive tool in regenerative medicine, with vast applications. They are easily obtained, hold multiple potency (21), and have been suggested for use in liver transplantation to prolong patient survival, with ADSC transplantation shown to promote liver recovery in mice with liver injury $(22,23)$. In the present study, hADSCs were successfully isolated from human adipose tissue and it was found that these cells were able to differentiate into hepatocyte-like cells following incubation in hepatocyte growing conditions, which was consistent with previous studies (24-27).

The hADSCs separated from the adipose tissue remained pluripotent and retained CD13 and CD90 stem cell surface markers. In addition they demonstrated the ability of adipogenic differentiation and osteogenic differentiation. These results suggested the potential of hADSCs for use in stem-cell-based therapies. Furthermore, ADSCs may be obtained repeatedly and in large quantities, through a convenient and safe procedure, thus presenting a practical alternative to BMSCs for clinical applications (28). Although BMSCs have the same biological properties as ADSCs, the numbers of hMSCs, obtained through marrow aspiration, decline with age (29). In addition, there is an age-related decline in overall BMSC differentiation potency, which poses a problem for their use in cell-based therapies. By contrast, the differentiation capacity of ADSCs is minimally affected by donor age (30).

The mechanism underlying hepatic ADSC differentiation remains to be fully elucidated. Previous studies have focused on the clinical and tissue engineering applications of ADSCs in regenerative medicine $(4,31)$, however, they have not investigated the capacity of ADSCs, as somatic cells, to retain multipotent differentiation functionality. Several of these studies have focussed on a description of the phenomenon, rather than examination of the mechanism (32).

Our previous study investigated the role of Cav-1 in human hepatocyte proliferation using Chang liver cells (CHL), and found that the downregulation of $\mathrm{Cav}-1$ induced biphasic change in growth rate, associated with AKT and MAPK signaling (33). In addition, Cav-1 has been shown to be involved in the progress of liver regeneration and liver development $(14,34,35)$. Previous results have also indicated that the expression of Cav-1 in the mouse liver increased in a time-dependent manner across developmental stages from natal to 3 months post-natal. However, whether Cav-1 has the same effect in the hepatic differentiation of hADSCs has received little attention. In the present study, hADSCs were successfully induced into hepatocyte-like cells, and the observed trend of expression of Cav-1 in the hADSCs during hepatic differentiation was the same as that observed in mouse liver cells during development, with undifferentiated hADSCs exhibiting low expression levels of Cav-1, which gradually increased as hepatic differentiation commences. This is not an unexpected result, as cholesterol and lipid metabolism are acquired during liver development $(36,37)$ and Cav-1 has been shown to be key in this process $(38,39)$. In addition, the present study found that Cav-1 knockdown was accompanied by a decrease in HNF1A and ALB, indicating that Cav-1 is essential in the hepatic differentiation process of hADSCs. Although it was observed that the expression of Cav-1 increased significantly on day 14 of hepatic differentiation, this expression level was low compared with those in Chang liver cells, suggesting that preservation of certain stem cell properties in the hADSCs.

Liver regeneration also requires hepatocyte proliferation, and the MAPK signaling pathway has a major role in this process (40). A previous study reported that the MAPK signaling pathway is important for ADSC transplantation into the damaged liver (41). The results of the present study demonstrated that p-MAPK significantly decreases during Cav- 1 knockdown, resulting in a subsequent decrease in the levels of $A L B$ and $H N F 1 A$, an indication of failed hepatic differentiation. Taken together, these results suggested that the Cav-1-MAPK signaling pathway is important in hADSC hepatic differentiation.

The present study showed for the first time, to the best of our knowledge, that Cav-1 is essential for hADSC hepatic differentiation, and the mechanism underlying this differentiation was further characterized. The results suggested that the regulation of Cav-1 and its associated pathway presents a promising avenue for further investigation, with the ultimate aim to increase the efficiency of hADSC hepatic differentiation for use in practical stem cell-based clinical applications.

\section{Acknowledgements}

This study was supported by the Chinese National Natural Science Foundation (grant nos. 81071009 and 81271412), the International S\&T Cooperation Project of the Ministry of S\&T of China, (grant no. 2010DFR30850), the Science and Technology Plan Projects in Liaoning Province (grant no. 201501522) and the Scientific Research Foundation for the Returned Overseas Chinese Scholars, State Education Ministry.

\section{References}

1. Glanemann M, Gaebelein G, Nussler N, Hao L, Kronbach Z, Shi B, Neuhaus P and Nussler AK: Transplantation of monocyte-derived hepatocyte-like cells (NeoHeps) improves survival in a model of acute liver failure. Ann Surg 249: 149-154, 2009. 
2. Hossein Aghdaie M, Geramizadeh B, Azarpira N Esfandiari E, Darai M, Rahsaz M, Nikeghbalian S and Malekhosseini SA: Hepatocyte isolation from unused/ rejected livers for transplantation: Initial step toward hepatocyte transplantation, the first experience from iran. Hepat Mon 13: e10397, 2013.

3. Forbes SJ and Alison MR: Regenerative medicine: Knocking on the door to successful hepatocyte transplantation. Nat Rev Gastroenterol Hepatol 11: 277-278, 2014.

4. Zheng MH, Ye C, Braddock $M$ and Chen YP: Liver tissue engineering: Promises and prospects of new technology. Cytotherapy 12: 349-360, 2010.

5. Williams AR and Hare JM: Mesenchymal stem cells: Biology, pathophysiology, translational findings and therapeutic implications for cardiac disease. Circ Res 109: 923-940, 2011.

6. Aurich H, Sgodda M, Kaltwasser P, Vetter M, Weise A, Liehr T, Brulport M, Hengstler JG, Dollinger MM, Fleig WE and Christ B: Hepatocyte differentiation of mesenchymal stem cells from human adipose tissue in vitro promotes hepatic integration in vivo. Gut 58: 570-581,2009.

7. Anzalone R, Lo Iacono M, Corrao S, Magno F, Loria T, Cappello F, Zummo G, Farina F and La Rocca G: New emerging potentials for human Wharton's jelly mesenchymal stem cells: Immunological features and hepatocyte-like differentiative capacity. Stem Cells Dev 19: 423-438, 2010.

8. Al Battah F, De Kock J, Vanhaecke T and Rogiers V: Current status of human adipose-derived stem cells: Differentiation into hepatocyte-like cells. ScientificWorldJournal 11: 1568-1581, 2011.

9. Zhu Y, Liu T, Song K, Fan X, Ma X and Cui Z: Adipose-derived stem cell: A better stem cell than BMSC. Cell Biochem Funct 26 664-675, 2008.

10. Huang SJ, Fu RH, Shyu WC, Liu SP, Jong GP, Chiu YW, Wu HS, Tsou YA, Cheng CW and Lin SZ: Adipose-derived stem cells: Isolation, characterization and differentiation potential. Cell Transplant 22: 701-709, 2013.

11. Ruiz JC, Ludlow JW, Sherwood S, Yu G, Wu X and Gimble JM: Differentiated human adipose-derived stem cells exhibit hepatogenic capability in vitro and in vivo. J Cell Physiol 225: 429-436, 2010.

12. Zhang X, Shen P, Coleman M, Zou W, Loggie BW, Smith LM and Wang Z: Caveolin-1 down-regulation activates estrogen receptor alpha expression and leads to 17beta-estradiol-stimulated mammary tumorigenesis. Anticancer Res 25: 369-375, 2005.

13. Wang XX, Wu Z, Huang HF, Han C, Zou W and Liu J: Caveolin-1, through its ability to negatively regulate TLR4, is a crucial determinant of MAPK activation in LPS-challenged mammary epithelial cells. Asian Pac J Cancer Prev 14: 2295-2299, 2013.

14. Frank PG and Lisanti MP: Caveolin-1 and liver regeneration: role in proliferation and lipogenesis. Cell Cycle 6: 115-116, 2007.

15. Fernández-Rojo MA, Gongora M, Fitzsimmons RL, Martel N, Martin SD, Nixon SJ, Brooks AJ, Ikonomopoulou MP, Martin S, Lo HP, et al: Caveolin-1 is necessary for hepatic oxidative lipid metabolism: Evidence for crosstalk between caveolin-1 and bile acid signaling. Cell Rep 4: 238-247, 2013.

16. Salem AF, Bonuccelli G, Bevilacqua G, Arafat H, Pestell RG, Sotgia F and Lisanti MP: Caveolin-1 promotes pancreatic cancer cell differentiation and restores membranous E-cadherin via suppression of the epithelial-mesenchymal transition. Cell Cycle 10: 3692-3700, 2011.

17. Li Y, Luo J, Lau WM, Zheng G, Fu S, Wang TT, Zeng HP, So KF, Chung SK, Tong Y, et al: Caveolin-1 plays a crucial role in inhibiting neuronal differentiation of neural stem/progenitor cells via VEGF signaling-dependent pathway. PLoS One 6: e22901, 2011.

18. Rossi S, Poliani PL, Cominelli M, Bozzato A, Vescovi R, Monti E and Fanzani A: Caveolin 1 is a marker of poor differentiation in Rhabdomyosarcoma. Eur J Cancer 47: 761-772, 2011.

19. Locke M, Windsor J and Dunbar PR: Human adipose-derived stem cells: Isolation, characterization and applications in surgery. ANZ J Surg 79: 235-244, 2009.

20. Bunnell BA, Flaat M, Gagliardi C, Patel B and Ripoll C: Adipose-derived stem cells: Isolation, expansion and differentiation. Methods 45:115-120, 2008.

21. Gimble JM, Katz AJ and Bunnell BA: Adipose-derived stem cells for regenerative medicine. Circ Res 100: 1249-1260, 2007.
22. Banas A, Teratani T, Yamamoto Y, Tokuhara M, Takeshita F, Osaki M, Kawamata M, Kato T, Okochi H and Ochiya T: IFATS collection: In vivo therapeutic potential of human adipose tissue mesenchymal stem cells after transplantation into mice with liver injury. Stem Cells 26: 2705-2712, 2008.

23. Banas A, Teratani T, Yamamoto Y, Tokuhara M, Takeshita F, Osaki M, Kato T, Okochi H and Ochiya T: Rapid hepatic fate specification of adipose-derived stem cells and their therapeutic potential for liver failure. J Gastroenterol Hepatol 24: 70-77, 2009.

24. Banas A, Teratani T, Yamamoto Y, Tokuhara M, Takeshita F, Quinn G, Okochi $\mathrm{H}$ and Ochiya T: Adipose tissue-derived mesenchymal stem cells as a source of human hepatocytes. Hepatology 46: 219-228, 2007.

25. Sgodda M, Aurich H, Kleist S, Aurich I, König S, Dollinger MM, Fleig WE and Christ B: Hepatocyte differentiation of mesenchymal stem cells from rat peritoneal adipose tissue in vitro and in vivo. Exp Cell Res 313: 2875-2886, 2007.

26. Chen X, Zhang S, Liu T, Liu Y and Wang Y: Maintenance of rat hepatocytes under inflammation by coculture with human orbital fat-derived stem cells. Cell Mol Biol Lett 17: 182-195, 2012.

27. Li X, Yuan J, Li W, Liu S, Hua M, Lu X and Zhang H: Direct differentiation of homogeneous human adipose stem cells into functional hepatocytes by mimicking liver embryogenesis. J Cell Physiol 229: 801-812, 2014.

28. Mimeault M and Batra SK: Recent progress on tissue-resident adult stem cell biology and their therapeutic implications. Stem Cell Rev 4: 27-49, 2008.

29. Stolzing A, Jones E, McGonagle D and Scutt A: Age-related changes in human bone marrow-derived mesenchymal stem cells: Consequences for cell therapies. Mech Ageing Dev 129: 163-173, 2008.

30. Shi YY, Nacamuli RP, Salim A and Longaker MT: The osteogenic potential of adipose-derived mesenchymal cells is maintained with aging. Plast Reconstr Surg 116: 1686-1696, 2005.

31. Mailey B, Hosseini A, Baker J, Young A, Alfonso Z, Hicok K, Wallace AM and Cohen SR: Adipose-derived stem cells: Methods for isolation and applications for clinical use. Methods Mol Biol 1210: 161-181, 2014.

32. Sterodimas A, de Faria J, Nicaretta B and Pitanguy I: Tissue engineering with adipose-derived stem cells (ADSCs): Current and future applications. J Plast Reconstr Aesthet Surg 63: 1886-1892, 2010.

33. Ren G, Liu Y, Wang XM, Zhao CH and Zou W: Role of caveolin-1 down-regulation by iRNA in human hepatocyte proliferation. Zhonghua Gan Zang Bing Za Zhi 16: 379-382, 2008 (In Chinese).

34. Fernández MA, Albor $\mathrm{C}$, Ingelmo-Torres $\mathrm{M}$, Nixon SJ, Ferguson C, Kurzchalia T, Tebar F, Enrich C, Parton RG and Pol A: Caveolin-1 is essential for liver regeneration. Science 313: $1628-1632,2006$.

35. Mayoral R, Fernández-Martínez A, Roy R, Boscá L and Martín-Sanz P: Dispensability and dynamics of caveolin-1 during liver regeneration and in isolated hepatic cells. Hepatology 46: 813-822, 2007.

36. Bosch M, Marí M, Herms A, Fernández A, Fajardo A, Kassan A, Giralt A, Colell A, Balgoma D, Barbero E, et al: Caveolin-1 deficiency causes cholesterol-dependent mitochondrial dysfunction and apoptotic susceptibility. Curr Biol 21: 681-686, 2011.

37. Baker $\mathrm{N}$ and Tuan RS: The less-often-traveled surface of stem cells: Caveolin-1 and caveolae in stem cells, tissue repair and regeneration. Stem Cell Res Ther 4: 90, 2013.

38. Frank PG, Pavlides S, Cheung MW, Daumer K and Lisanti MP: Role of caveolin-1 in the regulation of lipoprotein metabolism. Am J Physiol Cell Physiol 295: C242-C248, 2008.

39. Fernández-Rojo MA, Restall C, Ferguson C, Martel N, Martin S, Bosch M, Kassan A, Leong GM, Martin SD, McGee SL, et al: Caveolin-1 orchestrates the balance between glucose and lipid-dependent energy metabolism: Implications for liver regeneration. Hepatology 55: 1574-1584, 2012.

40. Campbell JS, Argast GM, Yuen SY, Hayes B and Fausto N: Inactivation of p38 MAPK during liver regeneration. Int J Biochem Cell Biol 43: 180-188, 2011.

41. Liang L, Ma T, Chen W, Hu J, Bai X, Li J and Liang T: Therapeutic potential and related signal pathway of adipose-derived stem cell transplantation for rat liver injury. Hepatol Res 39: 822-832, 2009. 